\title{
Prospective External Validation of the Clinical Effectiveness of an Emergency Department-Based Early Goal-Directed Therapy Protocol for Severe Sepsis and Septic Shock ${ }^{*}$
}

\author{
Alan E. Jones, MD, Anne Focht, RN, MSN, James M. Horton, MD, and Jeffrey A. Kline, MD
}

\begin{abstract}
Objective-To determine the clinical effectiveness of implementing early goal-directed therapy (EGDT) as a routine protocol in the emergency department (ED).

Methods-Prospective interventional study conducted over 2 years at an urban ED. Inclusion criteria included suspected infection, criteria for systemic inflammation, and either systolic BP $<90$ $\mathrm{mm} \mathrm{Hg}$ after a fluid bolus or lactate concentration $\geq 4 \mathrm{~mol} / \mathrm{L}$. Exclusion criteria were age $<18$ years, contraindication to a chest central venous catheter, and need for immediate surgery. We prospectively recorded preintervention clinical and mortality data on consecutive, eligible patients for 1 year when treatment was at the discretion of board-certified emergency physicians. We then implemented an EGDT protocol (the intervention) and recorded clinical data and mortality rates for 1 year. Prior to the first year, we defined a 33\% relative reduction in mortality (relative mortality reduction that was found in the original EGDT trial) to indicate clinical effectiveness of the intervention.
\end{abstract}

Results-We enrolled 79 patients in the preintervention year and 77 patients in the postintervention year. Compared with the preintervention year, patients in the postintervention year received significantly greater crystalloid volume $(2.54 \mathrm{~L}$ vs $4.66 \mathrm{~L}, \mathrm{p}<0.001)$ and frequency of vasopressor infusion $(34 \%$ vs $69 \%, \mathrm{p}<0.001)$ during the initial resuscitation. In-hospital mortality was 21 of 79 patients (27\%) before intervention, compared with 14 of 77 patients (18\%) after intervention (absolute difference, - 9\%; 95\% confidence interval, +5 to - $21 \%$ ).

Conclusions-Implementation of EGDT in our ED was associated with a 9\% absolute (33\% relative) mortality reduction. Our data provide external validation of the clinical effectiveness of EGDT to treat sepsis and septic shock in the ED.

\section{Keywords}

early goal-directed therapy; emergency department; resuscitation; sepsis; septic shock

sepsis has an in-hospital mortality rate of $30 \%$, equating to approximately 215,000 deaths annually. ${ }^{1-2}$ An estimated $50 \%$ of hospital sepsis cases originate in the emergency department (ED). ${ }^{3}$ New therapeutic strategies for sepsis have had little impact on the high mortality rate as evidenced by the finding that the average mortality rate attributable to sepsis in the last decade approximately equaled the mortality rates from the 1970 s. ${ }^{4}$ However, in the last 5 years,

\footnotetext{
*From the Department of Emergency Medicine (Drs. Jones and Kline), Clinical Care Management (Ms. Focht), and Department of Internal Medicine (Dr. Horton), Carolinas Medical Center, Charlotte, NC.

Copyright () 2007 by American College of Chest Physicians

Correspondence to: Alan E. Jones, MD, Assistant Director of Research, Department of Emergency Medicine, 1000 Blythe Blvd, MEB 304e, Carolinas Medical Center, Charlotte, NC 28203; e-mail: E-mail: alan.jones@ carolinas.org.

None of the authors have conflicts of interest to report.
} 
several therapeutic research innovations have shown promise for impacting the outcome of patients with sepsis. $5-7$

Among these innovations, the largest mortality benefit was reported by Rivers and colleagues 7 in a study of 263 patients enrolled in a randomized controlled trial in 2001 . Through a protocol-driven resuscitation strategy aimed at hemodynamic optimization in the ED, at the time of recognition of sepsis, Rivers et al ${ }^{7}$ were able to decrease absolute inhospital mortality rate by $16 \%$. Their strategy, termed early goal-directed therapy (EGDT), targets various end points of resuscitation in an algorithmic approach with the overarching goal of reversing sepsisinduced hypoperfusion. The results of this study led to a grade B recommendation for the routine use of EGDT in the ED for patients who present with severe sepsis and septic shock as a part of the Surviving Sepsis Campaign guidelines. These recommendations have been endorsed by 11 professional societies, including the American College of Emergency Physicians and Society of Critical Care Medicine. ${ }^{8}$ However, this approach has not been externally validated in prospective longitudinal study that measured the effect of the intervention of the EGDT protocol, used as published by Rivers et al. ${ }^{7}$ In the present study, we sought to determine the clinical effectiveness, quantified by predefined mortality and morbidity indicators, of patients treated with the protocol compared with patients treated prior to the protocol implementation.

\section{Materials and Methods}

\section{Study Design and Setting}

We performed a prospective before and after study of the clinical effectiveness, defined as the change in mortality caused by the intervention of an EGDT protocol for the early treatment of severe sepsis and septic shock in the ED. The protocol was implemented as a clinical pathway in the time frame diagrammed in Figure 1. This study was reviewed and approved by the institutional review board and privacy board of Carolinas Healthcare System.

All patients were enrolled in the ED at Carolinas Medical Center, an urban, 800-bed, teaching hospital with $>100,000$ patient visits per year. The ED is staffed at all times by emergency medicine resident physicians supervised by board-certified emergency medicine attending physicians. Prior to implementing the goal-directed resuscitation protocol, our ED did not follow written management protocol for patients with severe sepsis or septic shock.

\section{Treatment Protocol Development and Implementation}

A multidisciplinary team of ED, ICU, and infectious disease specialists, and ED and ICU nurses collaborated to implement the protocol and methods described by Rivers et al7 (Fig 2). Our protocol was the same but was delivered via two modifications: (1) it was executed by the physicians and nurses who were providing clinical care to the patient (Rivers et al provided additional physician staffing at the bedside); and (2) it was initiated in the ED, and care was subsequently transitioned to the ICU. The protocol was discontinued at the discretion of the admitting attending physician (Rivers et al continued care in the ED for $6 \mathrm{~h}$ ).

The goal-directed resuscitation protocol required monitoring central venous pressure (CVP) and central venous oxygen saturation $\left(\mathrm{Scvo}_{2}\right)$, which our preliminary data demonstrated were not performed in severe sepsis or septic shock patients in our ED prior to initiation of the protocol. Accordingly, 1 month was allocated for the education and training of participating healthcare providers. To minimize difficulties inherent to the transfer of care, we developed a standard goal-directed resuscitation order sheet and a nursing flow sheet to facilitate the patient's transfer to the ICU. 9 


\section{Study Subjects}

In both phases, eligible subjects were identified by board-certified emergency physicians in the ED, and inclusion criteria were identical for both phases: (1) age > 17 years; (2) suspected or confirmed infection; (3) two or more systemic inflammatory response syndrome criteria10: heart rate $>90$ beats $/ \mathrm{min}$; respiratory rate, $>20$ breaths $/ \mathrm{min}$; temperature, $>38^{\circ} \mathrm{C}$ or $<36^{\circ} \mathrm{C}$, WBC count $>12,000 / \mu$ Lor $<4,000 / \mu$ Lor $>10 \%$ bands; (4) systolic BP $<90 \mathrm{~mm} \mathrm{Hg}$ or mean arterial pressure $<65 \mathrm{~mm} \mathrm{Hg}([2 \times$ systolic $\mathrm{BP}+$ diastolic $\mathrm{BP}] / 3)$ after a $20 \mathrm{~mL} / \mathrm{kg}$ isotonic fluid bolus or anticipated need for ICU care and a serum lactate concentration $\geq 4.0 \mathrm{mmol} / \mathrm{L}$. Exclusion criteria in both phases were as follows: (1) age < 18 years; (2) need for immediate surgery (anticipated departure to the operating room in $<6 \mathrm{~h}$ and thus could not receive $6 \mathrm{~h}$ of therapy in the ED and/or ICU); and (3) absolute contraindication for a chest central venous catheter. Patients with do-not-resuscitate orders at the time of eligibility were eligible for treatment with the protocol only after discussion with the family and admitting attending physician, and if both agreed on aggressive medical treatment.

In the before phase, physicians were given pocket cards containing the inclusion and exclusion criteria. Patient data were entered in real time on a computer in the ED using a secure Webbased electronic collection form similar to that described by Kline et al. ${ }^{11}$ In the before phase, physicians were not aware of the plans for implementation of EGDT, and none had previously used the protocol described by Rivers et al. ${ }^{7}$

In the after phase, identification of an eligible patient triggered an alphanumeric page to the ED and ICU charge nurses, ED respiratory therapist, pharmacy, bed management, and both the protocol quality assurance nurse and physician. The page was meant to notify all potential clinical staff involved that the protocol was being initiated, and to accurately identify all protocol subjects. The charge nurses were notified in order to ensure smooth patient transition, the ED therapist was notified in the event that any respiratory needs were necessary (eg, assistance with initiation of mechanical ventilation), the pharmacy was notified in the event antimicrobial questions arose, and bed management was notified in order to begin the process of securing an ICU bed. The page did not require that any of these individuals respond to the patient's bedside. There was no bedside participation in the care of patients by any research personnel, there was no additional staffing of either the ED or ICU, there was no redistribution of clinical responsibilities of any of the staff, and there was no modification of either the ED or ICU physical facilities. A protocol quality assurance physician with experience in protocol execution (A.E.J.) was available by pager or telephone $(24 \mathrm{~h} / \mathrm{d})$ to answer questions about protocol execution. The participation of the quality assurance physician as a resource was voluntary, used infrequently by the treating physicians (6\% of cases) and he was never requested at the bedside. In all cases, the ED physicians and staff identified the patients, initiated goal-directed resuscitation, placed the central and arterial venous catheters, administered antimicrobial medications, and followed the protocol until a bed in the ICU was available for patient transfer. At the time of patient physical transfer from the ED to ICU, clinical care was transferred from the ED physicians and nurses to the admitting physicians and ICU nurses.

\section{Data Analysis and Outcomes}

The primary outcome was in-hospital mortality. We defined clinical effectiveness before the study began as a $33 \%$ relative mortality reduction over the course of 1 year after intervention. We chose this relative mortality reduction because it is the relative mortality reduction that was found in the original EGDT trial by Rivers et al. ${ }^{7}$ This design precluded a formal sample size estimate. 
Secondary outcomes were the hospital resources utilized including the mean number of both ICU and hospital days and the mean number of ventilator days. For both hospital and ICU days, if a patient spent any amount during the 24-h period of 1 day in the ICU or hospital, this was counted as a full day. The days of mechanical ventilation was defined as the number of days receiving mechanical ventilation in all patients receiving ventilation divided by number of patients receiving ventilation during the study period. We also recorded if any sepsis-specific therapies were administered, such as parenteral corticosteroids and activated protein $\mathrm{C}$. The Sequential Organ Failure Assessment score and Mortality in Emergency Department Sepsis (MEDS) scores were calculated on all patients at the time of identification. ${ }^{12-13}$

Continuous data are presented as mean $\pm \mathrm{SD}$, and when appropriate were compared for statistical differences using unpaired $t$ tests or Mann Whitney $U$ tests. Categorical data are reported as proportions rounded to the nearest whole number and associated 95\% confidence intervals (CIs), and where applicable tested for significance using $\chi^{2}$ or Fisher exact tests. The Kaplan-Meier survival estimates and log-rank test for comparison were used for time-toprimary outcome analysis. For all statistical tests, $\mathrm{p}<0.05$ was considered significant.

\section{Results}

We enrolled 157 patients: 79 in the before phase (August 1, 2004, to September 30, 2005) and 77 in the after phase (November 1, 2005, to October 31, 2006). In all 77 postintervention patients, ED physicians and staff identified the patients, initiated the goal-directed resuscitation protocol, placed the central venous catheter, administered antimicrobial medications, and followed the protocol until a bed in the ICU was available for patient transfer. Table 1 shows the demographics, comorbidities, clinical variables, severity of illness score, and source of suspected infections between the groups. Among the after-group patients, the mean duration of protocol execution from initiation to transfer from the ED was $2 \mathrm{~h}$ and $20 \mathrm{~min}$.

Table 2 compares the resuscitation interventions utilized in the initial $6 \mathrm{~h}$ of resuscitation between groups. Patients in the after group received a significantly greater crystalloid volume in the first $6 \mathrm{~h}$ as well as a significant increase in the frequency of the use of vasopressors and frequency of endotracheal intubation as compared to the before group. We observed no significant differences in the rate of packed RBC transfusion or dobutamine administration. Additionally, the time to antibiotic administration decreased significantly in the after group (99 $\min$ vs $142 \mathrm{~min}, \mathrm{p}=0.02$ ).

The primary outcome of in-hospital mortality was observed in 21 of 79 patients (27\%) in the before group and 14 of 77 patients (18\%) in the after group (difference, - 9\%; 95\% confidence interval, 5 to $-21 \% ; \chi^{2} \mathrm{p}=0.21$ ) [Table 3]. The Kaplan-Meier survival estimate were not significant between the groups for the primary outcome (log-rank test, $p=0.13$ ) [Fig 3]. The secondary outcomes are also shown in Table 3. Patients in the after group had an increase in mean ICU length of stay of 1.8 days and mean hospital length of stay of 1.2 days. The mean number of ventilator-days between the groups was identical.

\section{Discussion}

In this study, we document the clinical effectiveness of implementing an EGDT algorithm for the management of severe sepsis and septic shock in the ED. We found a 9\% absolute and 33\% relative mortality reduction, suggesting a number needed to treat (1/absolute mortality reduction) of approximately 11 persons. Furthermore, this mortality benefit was found among a group of patients with at least equal hemodynamic instability based on inspection of the systolic BP and sequential organ failure scores measured at enrollment. To our knowledge, this is the largest report evaluating the clinical effectiveness of EGDT that includes prospective 
identification and data collection on both before implementation (before) and after implementation (after) subjects since the sentinel report by Rivers et al. ${ }^{7}$

Previous full-length reports ${ }^{14-17}$ of implementing EGDT have found improved absolute mortality rates ranging from 9 to $28 \%$. These studies differ from the present report in that they were retrospective 17 used historical control subjects, ${ }^{14-15}$ investigated bundled therapies that included EGDT, ${ }^{14,17}$ had a primary outcome other than a hospital mortality end point, ${ }^{16}$ or were cohort studies without control (preimplementation) groups. ${ }^{18}$ The present report strengthens the conclusion of these previous reports by testing a protocol very similar to that in the original report by Rivers et al. ${ }^{7}$ We submit, however, that our slight modificationnamely the use of only ED staff to execute the protocol—would afford wider generalization of the protocol.

The control group in our study had a hospital mortality rate lower (27\%) than previous reports 7,14-17 of EGDT, which range from 29 to 53\%. Our before group patients had a lower severity of illness, evidenced by the slightly lower MEDS score, than the control groups of previous studies $14-15$ We speculate that the observed higher control group mortality rates in prior reports ${ }^{14-15,17}$ were secondary to systematic biases inherent to retrospective studies. Additionally, enrollment in a randomized controlled trials may have produced higher control mortality rates due to vigorous inclusion and exclusion criteria or inherent selection during the consent process. 7

Our data do not allow an inference as to whether one specific component of the protocol afforded treatment effect. Subjects in the intervention group received both a significantly larger volume of crystalloid infusion and a higher frequency of vasopressor administration. We observed no significant increase in frequency of packed RBC transfusions or dobutamine administration, consistent with previous reports. ${ }^{14-15,17}$ It might be argued that a Hawthorne effect causing heightened awareness by the clinical staff (physician and nurses) resulted in both earlier and more aggressive response to physiologic abnormalities. For example, time to initial antibiotic administration decreased in the after group (142 $\mathrm{min}$ vs $99 \mathrm{~min}$ ). Additionally, use of corticosteroids increased in the postintervention group (40\% vs $6 \%$ ), which may have impacted the results.

This study was designed to test the clinical effectiveness of EGDT as opposed to the efficacy of EGDT. An efficacy trial determines if a treatment does more good than harm when delivered under optimum conditions and in a uniform fashion. ${ }^{19}$ The efficacy of EGDT was established by Rivers et al. ${ }^{7}$ An effectiveness trial determines if a treatment does more good than harm when delivered under realworld conditions. ${ }^{19}$ Before starting this study, we chose the definition of effectiveness to be a $33 \%$ relative mortality reduction at 1 year after implementation. We chose this definition because it is the same relative mortality reduction found by Rivers et $\mathrm{al}^{7}$ in the original EGDT trial. We used 1 year as a surrogate to a numeric sample size; thus, we do not demonstrate statistically significant differences in mortality between the before and after groups.

When comparing the results of this study to that of Rivers et al, ${ }^{7}$ several points deserve comment. First, compared to the EGDT group in the study by Rivers et al, ${ }^{7}$ our after group had higher initial CVP and $\mathrm{Scvo}_{2}$ but a lower systolic $\mathrm{BP}$ and initial lactate concentrations than did the Rivers EGDT group. Additionally, we found a nonsignificant increase in the mean hospital length of stay (LOS) of 1.2 days $(p=0.30)$ and significant increase in the mean ICU LOS of 1.8 days $(\mathrm{p}<0.05)$ in the after group as compared to the before group. Rivers et al ${ }^{7}$ found a nonsignificant 0.2-day difference in hospital LOS between the control and EGDT groups and did not report mean ICU LOS. Similar to Rivers et al, ${ }^{7}$ we found no significant difference in the mean duration of mechanical ventilation between our groups $(\mathrm{p}=0.90)$. These 
data appear to indicate that we studied a less severely ill group of subjects than did Rivers et $\mathrm{al},{ }^{7}$ yet the protocol was still effective as indicated by the same relative mortality reduction found in both studies. Although we found no significant difference in hospital LOS, we significantly increased ICU LOS. This increase in resources utilized in the ICU is a potentially important finding that should be examined in future investigations. Finally, although we did not study a bundled protocol that included therapies other than EGDT (such as steroids and activated protein $\mathrm{C}$ ), our after group did receive these therapies more frequently ( $e g$, steroids) than did the before group. This is important inasmuch as these therapies were likely not routinely administered when the original EGDT was studied was performed prior to 2001 . $^{7}$ Thus, when interpreting the results of our study, it is important to consider this potentially important change in routine management over time.

This report has several limitations that warrant discussion. The first limitation is a lack of a randomized design; however, this study was intended to document the clinical effectiveness of implementing the protocol into routine clinical practice rather than replicate the original study. ${ }^{7}$ Second, as previously discussed, the sample size of the study does not permit conclusions to be drawn about the statistical differences in the mortality rate between the groups. Third, it is possible that a systematic inclusion bias influenced the results of the study ( $e g$, in the before arm, subjects may have been missed due to underrecognition of sepsis as the etiology of illness; in the after group, patients may have been admitted with sepsis and not treated with the resuscitation protocol). Fourth, as previously mentioned, early antibiotics or increased use of steroids may have influenced the mortality difference between the groups. Also, it is possible that therapies administered after the EGDT period (eg, during the first 72 $\mathrm{h}$ of ICU care) contributed to the improved outcome. Finally we did not quantify, explore, or exclude protocol deviations because this study was designed to observe the effectiveness of the protocol in a clinical, nonresearch setting. Our intention was not to quantify the number or instances where all the goals of EGDT were achieved but rather to quantify the effect of $6 \mathrm{~h}$ of therapy had on patient outcome.

\section{Conclusion}

In this investigation we demonstrate the clinical effectiveness, quantified by a $9 \%$ absolute and $33 \%$ relative mortality reduction, of implementing EGDT in ED patients with severe sepsis and septic shock. Our data suggest a number needed to treat of approximately 11 persons.

\section{ACKNOWLEDGMENT}

We wish to thank all of the emergency medicine and critical care physicians and nurses, whose tireless efforts and dedication to the care of sepsis patients at our institution is impeccable. Additionally, we would like to thank the following members of our institutional sepsis task force for their efforts in operationalizing this protocol: Nancy Gritter, MD; Daniel Howard, MD; Toan Huynh, MD; Mike Johnson, MD; Eddie Leonhardt, RN; Lewis McCurdy, MD; Stephen Moore, MD; Richard Rissmiller, Jr, MD; Thomas Stern, MD; Joan Settlemyer, Pharm D; Sarah Turner, NP; Will Miles, MD. We would also like to thank Susan Freeman, John Marx, MD, Barbara DeSilva, and Kathleen Benfield and for administrative support of this initiative.

The author's institution received a $\$ 3,000$ educational grant from Edwards Lifesciences for continuing nursing and physician education. Dr Jones is supported by grant K23GM076652- 01A1 from the National Institute of General Medical Sciences/National Institutes of Health.

\section{Abbreviations}

CVP, central venous pressure; ED, emergency department; EGDT, early goal-directed therapy; LOS, length of stay; MEDS, Mortality in Emergency Department Sepsis; $\mathrm{Scvo}_{2}$, central venous oxygen saturation. 


\section{References}

1. Angus DC, Linde-Zwirble WT, Lidicker J, et al. Epidemiology of severe sepsis in the United States: analysis of incidence, outcome, and associated costs of care. Crit Care Med 2001;29:1303-1310. [PubMed: 11445675]

2. Kochanek, KD.; Smith, B. National vital statistics report. In: Centers for Disease Control and Prevention., editor. Deaths: preliminary data for 2002. Vol. Vol 52. US Department of Health and Human Services, National Vital Statistics Report; Hyattsville, MD: 2004. p. 1-32.

3. Strehlow MC, Emond SD, Shapiro NI, et al. National study of emergency department visits for sepsis, 1992 to 2001. Ann Emerg Med 2006;48:326-331. [PubMed: 16934654]

4. Centers for Disease Control and Prevention. Current trends increase in national hospital discharge survey rates for septicemia - United States, 1979-1987. MMRW Morb Mortal Wkly Rep 1991;39:3134.

5. Bernard GR, Vincent JL, Laterre PF, et al. Efficacy and safety of recombinant human activated protein C for severe sepsis. N Engl J Med 2001;344:699-709. [PubMed: 11236773]

6. Annane D, Sebille V, Charpentier C, et al. Effect of treatment with low doses of hydrocortisone and fludrocortisone on mortality in patients with septic shock. JAMA 2002;288:862-871. [PubMed: 12186604]

7. Rivers E, Nguyen B, Havstad S, et al. Early goal-directed therapy in the treatment of severe sepsis and septic shock. N Engl J Med 2001;345:1368-1677. [PubMed: 11794169]

8. Dellinger RP, Carlet JM, Masur H, et al. Surviving sepsis campaign guidelines for management of severe sepsis and septic shock. Crit Care Med 2004;32:858-873. [PubMed: 15090974]

9. Shapiro NI, Howell M, Talmor D, et al. A blueprint for a sepsis protocol. Acad Emerg Med 2005;12:352-359. [PubMed: 15805328]

10. Bone RC, Balk RA, Cerra FB, et al. Definitions for sepsis and organ failure and guidelines for the use of innovative therapies in sepsis: the ACCP/SCCM Consensus Conference Committee; American College of Chest Physicians/Society of Critical Care Medicine. Chest 1992;101:1644-1655. [PubMed: 1303622]

11. Kline JA, Johnson CL, Webb WB, et al. Prospective study of clinician-entered research data in the emergency department using an internet-based system after the HIPAA privacy rule. BMC Med Inform Decis Mak 2004;4:17. [PubMed: 15479471]

12. Vincent JL, Moreno R, Takala J, et al. The SOFA (Sepsisrelated organ failure assessment) score to describe organ dysfunction/failure. Intensive Care Med 1996;22:707-710. [PubMed: 8844239]

13. Shapiro NI, Wolfe RE, Moore RB, et al. Mortality in emergency department sepsis (MEDS) score: a prospectively derived and validated clinical prediction rule. Crit Care Med 2003;31:670-675. [PubMed: 12626967]

14. Shapiro NI, Howell MD, Talmor D, et al. Implementation and outcomes of the Multiple Urgent Sepsis Therapies (MUST) protocol. Crit Care Med 2006;34:1025-1032. [PubMed: 16484890]

15. Trzeciak S, Dellinger RP, Abata NL, et al. Translating research to clinical practice: a 1-year experience with implementing early goal-directed therapy for septic shock in the emergency department. Chest 2006;129:225-235. [PubMed: 16478835]

16. Micek ST, Roubinian N, Heuring T, et al. Before-after study of a standardized hospital order set for the management of septic shock. Crit Care Med 2006;34:2707-2713. [PubMed: 16943733]

17. Kortgen A, Niederprum P, Bauer M. Implementation of an evidence-based "standard operating procedure" and outcome in septic shock. Crit Care Med 2006;34:943-949. [PubMed: 16484902]

18. Nguyen HB, Corbett SW, Steele R, et al. Implementation of a bundle of quality indicators for the early management of severe sepsis and septic shock is associated with decreased mortality. Crit Care Med 2007;35:1105-1112. [PubMed: 17334251]

19. Flay BR. Efficacy and effectiveness trials (and other phases of research) in the development of health promotion programs. Prev Med 1986;15:451-474. [PubMed: 3534875] 
$8 / 1 / 2004$

Pre-intervention phase begins
$10 / 1 / 2005$

Begin EGDT protocol education In ED and ICU
$10 / 31 / 2005$

End EGDT education and protocol implemented as standard-of-care
$11 / 1 / 2006$

Post-intervention phase ends

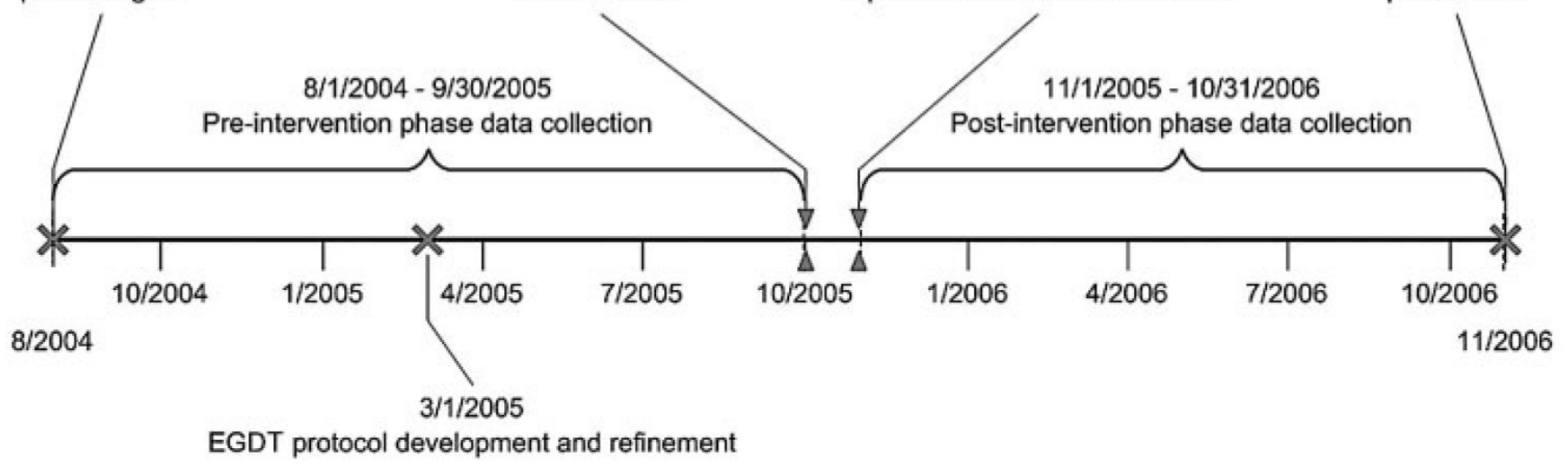

Figure 1.

Timeline of data collection and protocol implementation. 


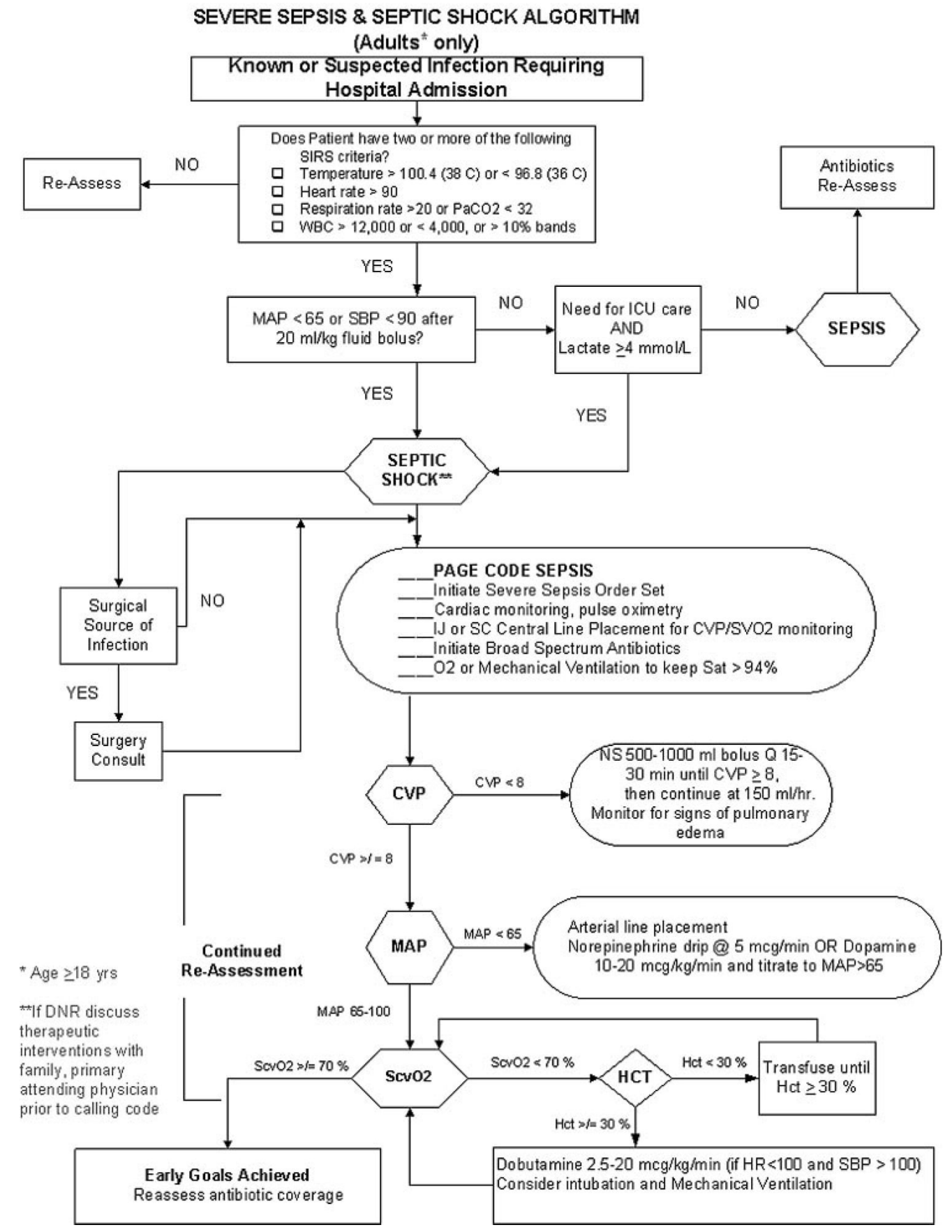

Figure 2.

Goal-directed resuscitation algorithm. SIRS $=$ systemic inflammatory response syndrome; $\mathrm{MAP}=$ mean arterial pressure; $\mathrm{SBP}=$ systolic $\mathrm{BP} ; \mathrm{IJ}=$ internal jugular; $\mathrm{SC}=$ subclavian; $\mathrm{HCT}$ $=$ hematocrit; $\mathrm{HR}=$ heart rate; $\mathrm{NS}=$ normal saline solution; $\mathrm{DNR}=$ do not resuscitate. 


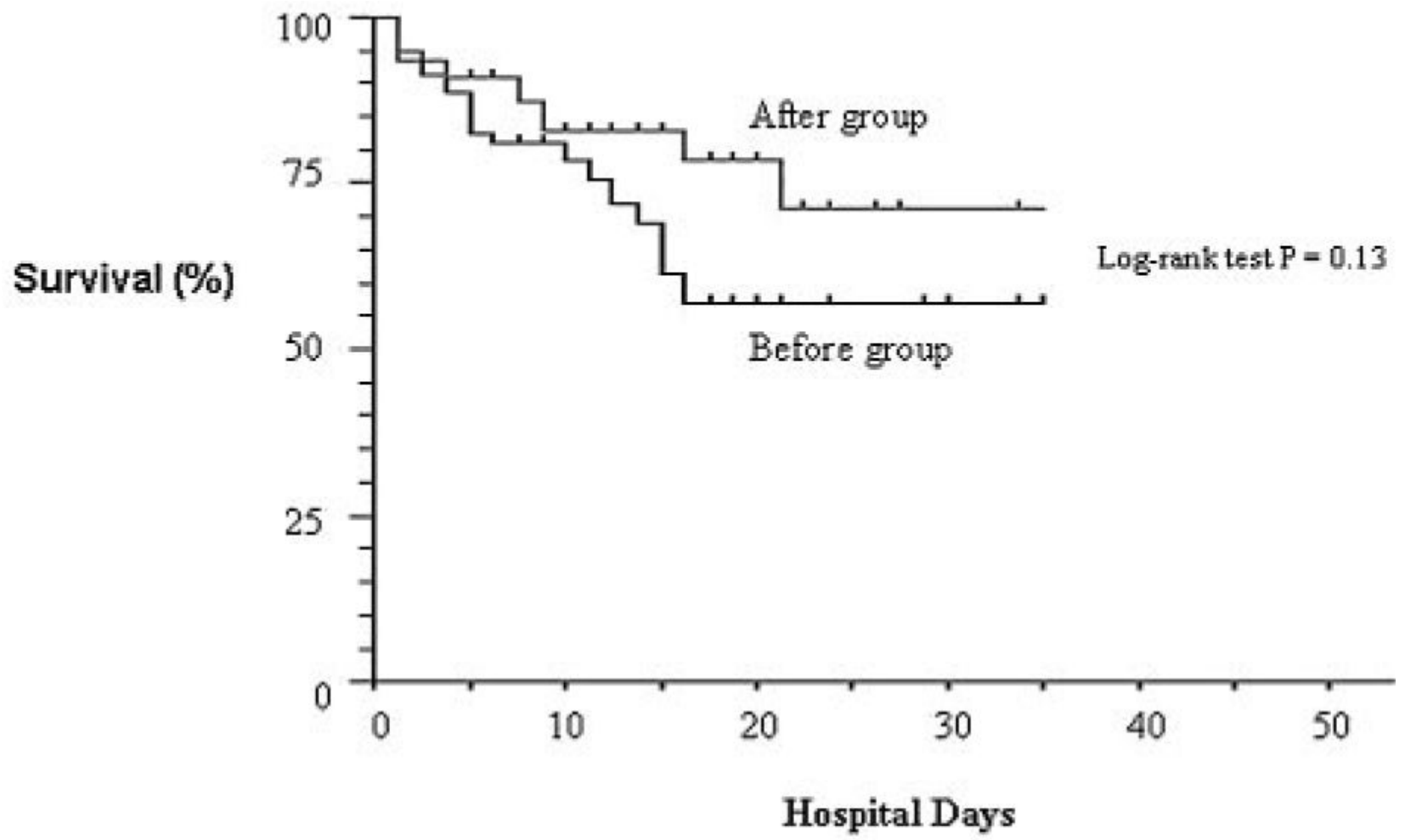

Figure 3.

Kaplan-Meier survival curve comparing survival of patients in the pre-EGDT intervention (before phase) and post-EGDT intervention (after group). This curve represents truncated data so that absolute survival is not observed on the plot but rather the survival in relation to hospital LOS. The time points represent the number of patients that remain in the hospital and are at risk of death. 
Table 1

Patient Demographics, Clinical Characteristics, and Physiologic Measurements*

\begin{tabular}{|c|c|c|}
\hline Variables & Before Group $(n=79)$ & After Group $(n=77)$ \\
\hline Age, yr & $57.6 \pm 15.6$ & $57.6 \pm 17.9$ \\
\hline \multicolumn{3}{|l|}{ Race } \\
\hline White & $40(51)$ & $46(60)$ \\
\hline Black & $38(48)$ & $27(35)$ \\
\hline \multicolumn{3}{|l|}{ Gender } \\
\hline Male & $47(59)$ & $48(62)$ \\
\hline Female & $32(41)$ & $29(38)$ \\
\hline \multicolumn{3}{|l|}{ Comorbitlities } \\
\hline Diabetes mellitus & $23(29)$ & $22(29)$ \\
\hline COPD & $12(15)$ & $17(22)$ \\
\hline HIV & $8(10)$ & $5(7)$ \\
\hline End-stage renal disease & $25(32)$ & $7(9)$ \\
\hline Cancer & $9(1)$ & $16(21)$ \\
\hline Organ transplant & $3(4)$ & $1(1)$ \\
\hline Indwelling vascular line & $7(9)$ & $11(14)$ \\
\hline Nursing home resident & $18(23)$ & $20(26)$ \\
\hline Do not resuscitate & $5(6)$ & $3(4)$ \\
\hline \multicolumn{3}{|l|}{ ED vital signs } \\
\hline Lowest systolic BP, mm Hg & $86 \pm 22.2$ & $72 \pm 12.3$ \\
\hline Highest pulse rate, beats/min & $118 \pm 26.7$ & $122 \pm 26.3$ \\
\hline Highest respiratory rate, breaths/min & $26 \pm 9.3$ & $30 \pm 10.2$ \\
\hline Highest temperature, ${ }^{\circ} \mathrm{C}$ & $38 \pm 2.9$ & $38 \pm 2.7$ \\
\hline Lowest oxygen saturation, $\%$ & $94 \pm 6.0$ & $92 \pm 7.9$ \\
\hline Highest CVP, mm Hg & & $7 \pm 4.5$ \\
\hline Highest CVP, mm Hg & & $14 \pm 5.9$ \\
\hline Highest $\mathrm{Scvo}_{2}, \%$ & & $69 \pm 12.7$ \\
\hline Highest $\mathrm{Scvo}_{2}, \%$ & & $81 \pm 8.5$ \\
\hline ED SOFA score & $5.3 \pm 3.3$ & $7.2 \pm 3.1$ \\
\hline ED MEDS score & $9.7 \pm 3.3$ & $11.1 \pm 3.4$ \\
\hline Lactate level, $\mathrm{mmol} / \mathrm{L}^{\dagger}$ & $4.8 \pm 3.4$ & $3.6 \pm 2.6$ \\
\hline \multicolumn{3}{|l|}{ Suspected source of infection } \\
\hline Pulmonary & $25(32)$ & $33(43)$ \\
\hline Urinary tract & $21(27)$ & $24(31)$ \\
\hline Intra-abdominal & $14(18)$ & $11(14)$ \\
\hline Skin/soft tissue & $16(20)$ & $13(17)$ \\
\hline Blood (bacteremia) & $3(4)$ & $8(13)$ \\
\hline Unknown & $12(15)$ & $8(10)$ \\
\hline
\end{tabular}

* Data are presented as mean \pm SD or No. $(\%)$. SOFA $=$ sequential organ failure assessment.

${ }^{\dagger}$ Lactate concentration was only available in 33 of 79 before-group patients and 66 of 79 after-group patients. 
${ }^{\ddagger}$ Some patients had more than one suspected source; thus, the total is $>100 \%$. 
Table 2

Resuscitation Interventions Utilized in the Initial $6 \mathrm{~h}^{*}$

\begin{tabular}{|c|c|c|c|}
\hline Interventions & Before Group $(n=79)$ & After Group $(n=77)$ & p Value ${ }^{\dagger}$ \\
\hline Endotracheal intubation & $7(9)$ & $27(35)$ & $<0.001$ \\
\hline Crystalloid volume, L & $2.54 \pm 2.4$ & $4.66 \pm 1.8$ & $<0.001$ \\
\hline Vasopressor administration & $27(34)$ & $53(69)$ & $<0.001$ \\
\hline Dobutamine administration & $1(1)$ & $2(3)$ & 0.61 \\
\hline Packed RBC transfusion & $1(1)$ & $4(5)$ & 0.21 \\
\hline \multicolumn{4}{|l|}{ Other } \\
\hline Time to initial antibiotics, min & $142 \pm 117$ & $99 \pm 72$ & 0.02 \\
\hline Steroid administration ${ }^{*}$ & $5(6)$ & $31(40)$ & $<0.001$ \\
\hline Activated protein $C^{\dagger}$ & $3(4)$ & $3(4)$ & 0.99 \\
\hline
\end{tabular}

* Data are presented as No. $(\%)$ or mean \pm SD.

${ }^{\dagger}$ Statistical analysis included t tests, Mann Whitney $U, \chi^{2}$, and Fisher exact tests as appropriate.

${ }^{*}$ Sepsis-specific variables that were followed but were not a part of the EGDT intervention protocol. Activated protein $\mathrm{C}$ was never administered in the ED or during the initial $6 \mathrm{~h}$ of therapy. Steroid administration was at the discretion of the attending physician in the ED or ICU but was not a part of the EGDT protocol. 
Table 3

Patient Primary and Secondary Outcomes

\begin{tabular}{lccc}
\hline Outcomes & Before Group $(\mathbf{n}=$ & After Group $(\mathbf{n}=\mathbf{7 7})$ & Difference \\
\hline In-hospital mortality, No. (\%) & $21(27)$ & $14(18)$ & $7(9)$ \\
Hospital resources utilized & & & \\
Mean ICU LOS, d & 2.0 & 3.8 & 1.8 \\
Mean hospital LOS, d & 7.9 & 9.1 & 1.2 \\
Mean ventilator days, d & 3.5 & 3.5 & 0 \\
\hline
\end{tabular}

